\title{
Tight junctions as regulators of tissue remodeling
}

Maria S. Balda ${ }^{1}$ and Karl Matter ${ }^{1}$

${ }^{1}$ Department of Cell Biology, UCL Institute of Ophthalmology, University College London, London, United Kingdom

Address for correspondence:

Maria S. Balda

Department of Cell Biology

UCL Institute of Ophthalmology

University College London

11-43 Bath Street

London EC1V 9EL United Kingdom

m.balda@ucl.ac.uk

T - 4402076086861 / F - 4402076084034

Words 2383 


\section{Abstract}

Formation of tissue barriers by epithelial and endothelial cells requires neighbouring cells to interact via intercellular junctions, which includes tight junctions. Tight junctions form a semipermeable paracellular diffusion barrier and act as signalling hubs that guide cell behaviour and differentiation. Components of tight junctions are also expressed in cell types not forming tight junctions, such as cardiomyocytes, where they associate with facia adherens and/or gap junctions. This review will focus on tight junction proteins expressed in epithelial and endothelial cells, and their importance in tissue homeostasis and remodelling with a particular emphasis on what we have learned from animal models and human diseases.

\section{Introduction}

Cells interact with their neighbours and the underlying extracellular matrix via specialized protein complexes that mediate adhesion, maintain structure and transmit information to the cell interior about the environment. This information is essential for different aspects of tissue functions as well as for physiological and pathological remodelling. At the lateral membrane of epithelia and endothelia, junctional complexes include tight junctions (TJ) and adherens junctions (AJs), two adhesion complexes that are structurally and functionally intertwined (Fig. 1). TJ form regulatable semipermeable paracellular barriers that control paracellular diffusion of solutes according to size and charge, and function as fences that restrict lipid diffusion between the apical and basolateral membrane domains (Fig. 2). TJ also act as signalling hubs that guide epithelial proliferation, death, polarisation and differentiation. TJ-associated transmembrane proteins interact with different components of the TJ-associated cytoplasmic plaque. Although not fully understood, these interactions result in a protein network that controls multiple cell functions involved in tissue remodelling such as junctional dynamics, cell migration, proliferation and gene expression. TJ structure, composition and function have recently been reviewed [1,2]. Here, we will focus on $T J$ and their contributions to tissue remodelling with an emphasis on transmembrane proteins and RhoGTPase signalling.

\section{Transmembrane proteins of $\mathrm{TJ}$}

TJ contain a complex set of transmembrane proteins that includes the tetraspan proteins of the claudin family and occludin-related Marvel domain porteins, as well as multiple adhesion proteins with immunoglobulin like domains. In vitro and in vivo studies have linked these proteins to different types of physiological and pathological examples of tissue remodeling (Table 1).

\section{Marvel domain proteins}

TJ contain three Marvel domain proteins: occludin, tricellulin or MarvelD2, and MarvelD3. MARVEL domains are motifs based on four transmembrane helices and are found in proteins involved in membrane-membrane aposition. Although the three proteins are related, they have distinct functions. Occludin knockout mice have a complex phenotype, which includes hyperplasia and chronic inflammation of the gastric epithelium, calcification in the brain, testicular atrophy, loss of cytoplasmic granules in striated duct cells of the salivary gland, and thinning of compact bones. The underlying mechanisms are not known. However, manipulation of occludin in culture affects the junctional barrier functions with its $\mathrm{N}$-terminal domain being important for the regulation of neutrophil transmigration. 
Occludin depletion also inhibits Rho activation required for extrusion of apoptotic cells from monolayers. It is thus possible that defects in leukocyte transmigration and cell extrusion lead to chronic inflammation and hyperplasia of epithelial tissues in vivo.

Occludin depletion leads to junctional remodelling with tricellulin, normally concentrated at tricellular corners, becoming more equally distributed along the cell periphery. Tricelllulin is essential for sealing tricellular corners and for hearing in human and mice; hence, occludin deficiency in mice also leads to deafness as the normal tricellulin localization is disrupted in the hair cells of the inner ear [3]. The underlying mechanisms are not well understood. However, tricellulin has recently been shown to regulate RhoGTPase signalling by recruiting the guanine-nucleotide-exchange factor (GEF) Tuba (ARHGEF36) and, thereby, modulating Cdc42 activation and tension generated at tricellular corners[4]. Whether the Tuba/tricellulin interaction is indeed related to hair cell degeneration and deafness is unknown.

The third Marvel protein, MarvelD3 regulates cell migration and proliferation in cells in culture, and MarvelD3 overexpression in pancreatic cancer cells inhibits tumour growth in vivo in mouse xenographs [5]. MarvelD3 modulates JNK activation by inhibiting MEKK1, an upstream component of the JNK pathway. This regulatory function is also important during osmotic stress-induced cytoskeletal remodelling, as MarvelD3-mediated tuning of JNK signalling is required to maintain junctional integrity and support cell survival. Further studies will need to address the role of MarvelD3 in tissue remodelling in vivo.

\section{Claudins}

Claudins are part of the paracellular diffusion barrier and mediate ion-selective paracellular diffusion by constituting conductive paracellular pores [6]. Knockout studies in mice support this role and report phenotypes related to barrier formation and paracellular ion diffusion. Barrier defects often lead to inflammatory responses and, consequently, tissue remodelling. Examples include knockouts of claudin-2, which leads to increased colorectal inflammation and gallstones; claudin-4, which provokes acute lung inflammation; and claudin-7, which induces intestinal inflammatory remodelling (Table 1) [7-9]. Given the barrier defects, however, it is generally assumed that the inflammation is an indirect response caused by increased tissue permeability rather than reflecting a direct role in inflammatory signalling. An exception is claudin-18. Although its deletion also leads to lung remodelling, it is also required to activate NF- $B$ in response to RANKL-stimulated osteoclast differentiation $[4,10-12]$

A different example is provided by claudin-1. Claudin-1 (-/-) mice have an abnormal skin structure and function. Claudin-1 is expressed in the stratum granulosum of the skin and skin remodelling is though to be due to changes in stratum granulosum composition, leading to aberrant stratum corneum structure and barrier function [13]. In humans, claudin-1 has been linked to ichthyosis and neonatal sclerosing cholangitis, diseases with skin and liver defects. The molecular mechanisms involved remain to be determined.

\section{JAMs}

JAMs are adhesion proteins with immunoglobulin like domains and have been linked to inflammation, angiogenesis and atherosclerosis. For example, the colonic mucosa of JAM-A-deficient mice shows increased claudin-10 and -15 expression, increased paracellular permeability, leukocyte infiltration and lymphoid aggregates, and, consequently, these mice are more susceptible to experimentally induced colitis [14]. In endothelia, JAM-A regulates angiogenesis and recruitment of monocytes to injured vessel walls, a process linked to atherosclerosis [15-17]. Thus, JAM-A seems to 
4

be involved in different signalling pathways and process involved in epithelial and endothelial remodelling but the responsible molecular mechanism(s) are not well understood.

The coxsackievirus and adenovirus receptor (CAR) belongs to the JAM superfamily and functions in the pathogenesis of coxsackievirus infection in vivo [18]. CAR is associated with heart intercalated disc adherens junctions, as well as lymphatic and epithelial TJ. CAR is essential for normal cardiac development, regulates proliferation and differentiation of cardiomyocytes, and adhesion of lymphatic endothelial cells [19]. Studies with postnatal mice further revealed that complex formation of CAR with connexin 45 is essential for electrical conduction between atrium and ventricle, and normal levels beta-catenin and ZO-1 at heart intercalated discs. Thus, junctional adhesion molecules perform essential functions in different cell types and can form functional complexes with other junctional proteins.

\section{The cytoplasmic plaque}

The TJ transmembrane proteins interact with a cytoplasmic plaque that links them to the cytoskeleton and signalling mechanisms that guide junction assembly and regulate cell behaviour. The cytoplasmic plaque is formed by multiple adaptor and scaffolding proteins (e.g., ZO-1/2/3); different types of signalling components such as GTP-binding proteins, protein kinases and phosphatases; as well as transcriptional and post-transcriptional regulators [20,21]. Several of the TJ associated plaque proteins exhibit dual localization at TJs and in the nucleus (e.g. ZONAB, cdk4, symplekin, ZO-2, YAP) linking $\mathrm{TJ}$ to the regulation of cell proliferation and gene expression. How cytoplasmic plaque proteins interact with transmembrane TJ proteins and how these interactions result in the control of TJ functions is not well understood; hence, we will only make occasional reference to transmembrane proteins when functionally relevant interactions are known.

\section{Rho GTPases and actomyosin regulation}

Tissue remodeling and TJ have been linked to regulators of Rho signalling involved in actin organisation, cell proliferation, gene expression and differentiation[22-24]. We are starting to understand how different GEFs and GAPs (GTPase-activating proteins) for different monomeric GTPases affect tissue remodelling, and are recruited and regulated by junctional adaptor proteins.

RhoA signalling - Three RhoA GEFs have been linked to TJ: ARHGEF2, 11 and 18. ARHGEF2/GEF-H1/Lfc regulates cell proliferation and cytoskeletal remodelling induced by different signalling pathways [25-27]. GEF-H1/LfC interacts with cingulin, a junctional adaptor, which inhibits the GEF by junctional sequestration and, thereby, attenuates cell proliferation. JACOP/paracingulin, a close relative of cingulin, seems to work redundantly in GEF-H1 regulation. GEF-H1 is inactive at TJ and, if activated in response growth factors or cell stress, mediates activation of RhoA signalling throughout the cell and activation of $Z O N A B$, a multifunctional protein that regulates transcriptional and posttranscriptional gene expression and, thereby, cell proliferation and survival [28,29]. In contrast, p114RhoGEF/ARHGEF18 is active at TJ and forms a complex with myosin II, Rock II and cingulin to drive junctional RhoA signalling and actomyosin contractility [30]. Its activity is hence important for a range of dynamic processes including junction assembly, migration of epithelial sheets and tumour cell invasion, as well as epithelial 3D morphogenesis [30,31]. p114RhoGEF also binds Lulu2, which is thought to be important during epithelial morphogenesis and apical constriction [32]. The third junctional RhoA GEF is ARHGEF11/PDZ-RhoGEF and is recruited to TJ by ZO-1 [33]. ARHGEF11 has recently been linked to insulin signalling in mice, suggesting that it may link TJ to regulation of metabolic pathways [34]. 
Rac and Cdc42 signalling - TJs have been also linked to regulation of Rac and Cdc42. Tuba, a Cdc42 GEF, interacts with ZO-1 and tricellulin; and Tuba depletion alters the configuration of cell junctions, resulting in a curved and slack appearance [4]. Tuba depletion leads to multilumen formation and defective spindle orientation in 3D cultures [35]. However, it has not known whether this reflects junctional Tuba functions. JACOP/ paracingulin not only interacts with GEF-H1 but also Tiam1, a Rac GEF, influencing actin remodelling during junctional assembly [36]. Junctional accumulation of cingulin and JACOP are also required for downregulation of Rac1 activity through the recruitment of MgcRacGAP [37]. Another junctional GAP important for Rac and Cdc42 signalling is SH3BP1, which is important for spatial control of Cdc42 activity [31]. SH3BP1 forms a complex with JACOP, CD2AP, and Capz, an actin capping protein, resulting in the formation of a dual activity signalling complex that guides actin remodelling by controlling RhoGTPase activity and actin polymerization.

\section{ZO proteins}

ZO-1 is a core TJ protein that binds to many cytosolic and transmembrane components, as well as F-actin [38]. The importance ZO-1 for the actomyosin cytoskeleton in epithelial cells remains controversial, as depletion has been linked to both increased and decreased junctional actomyosin [39]. Similarly, conflicting results have been reported for the importance of ZO-1 for junction formation and epithelial morphogenesis [39-41]. The effects seem to depend on the cell types analysed, and the level and method of downregulation (i.e., knockdown versus knockout, constitutive versus conditional depletion) $[39,40]$. As these in vitro experiments do not yet allow a firm conclusion about the role of ZO-1, it has been proposed that ZO-1 and its close homologue ZO-2 function redundantly. However, this is not compatible with in vivo experiments demonstrating that ZO-1 and ZO-2 are independently essential for normal embryonic development.

ZO-1-deficient embryos have defects in vascular development in the yolk sac, and the molecular mechanisms by which ZO-1 regulates endothelial junction formation and angiogenic remodelling is starting to be understood. ZO-1 depletion in primary endothelial cell cultures induces stress fibres and Rho activation along the basal membrane, leading to reduced tension on VE-cadherin-based adherens junctions, reduced cell migration, and defective barrier formation [41]. In vivo, this results in inhibition of FGF-2-induced angiogenesis. An important role of ZO-1 is the spatial organisation of tension acting on sites of adhesion. Its depletion leads to a redistribution of active myosin II and mechanotransducers like vinculin from junctions to stress fibres and focal adhesions, respectively. The redistribution of mechanotransducers is ROCK-dependent and can be phenocopied by JAM-A, JACOP, or p114RhoGEF (ARHGEF18) down-regulation [41]. It thus seems that ZO-1 regulates

junction formation by stimulating RhoA-activated junctional tension to balance tension generated along the basal membrane. Such a role for ZO-1 is also supported by the function of its homologue in C. elegans, zoo-1, which is required during elongation to prevent rupture of epidermal cell-cell junctions [42]. Similarly, the Drosophila homologue Polychaetoid localizes at AJ and regulates embryonic morphogenesis by regulating the actin cytoskeleton [43].

\section{Cell polarity and apical differentiation}

TJ-associated signalling mechanisms are important drivers of cell polarisation. The TJ polarity complexes were original identified in Drosophila (Crumbs/Pals1/Patj) and C. elegans (Par3/Par6/atypical PKC). The evolutionarily conserved Rac/Cdc42 effector PAR3/PAR6/aPKC complex is required for the formation of distinct tight and adherens junctions, and epithelial and 
endothelial morphogenesis in tissue culture $[44,45]$. These in vitro data are supported by in vivo studies, demonstrating that Par3 knockout in mice results in defective apical remodelling in epicardial cysts during cardiac morphogenesis. The PAR3/PAR6/aPKC complex cooperates with the Crumbs complex to guide apical polarisation. Of the three vertebrate Crumbs homologues, Crb3 is expressed in epithelia and associated with TJ. Deletion of Crb3 in mouse leads to death after birth and pathological remodelling in the kidney, lungs and intestine. It is thought that these defects are due to the role of Crb3 in polarity signalling, modulation of the cytoskeleton via associated proteins such as ezrin [46], and by coupling cell density sensing to Hippo-dependent control of the TGF- $\beta$-SMAD pathway [47]. Regulation of Cdc42 is tightly controlled during this process by two junctional GEFs: Ect2 stimulating Cdc42 activation during junction formation; and, once junctions have formed, Dbl3, which is recruited by ezrin to the apical pole, driving polar Cdc42 activation and apical morphogenesis [48].

In endothelia, the RhoA GEF Syx has been linked to the Crumbs complex and junction formation in mice and zebrafish. Syx is recruited to junctions by Mupp1, a close relative of Patj and part of the Crumbs polarity complex, and promotes junction integrity by activating the RhoA effector Diaphanous. Localization of Syx is regulated by two antagonistic mechanisms: VEGF causes translocation of Syx from cell junctions and Ang1 stabilises Syx at the junction [49]. Syx redistribution requires the Rab GTPase Rab13, which is in agreement with Rab13 knockdown experiments in zebrafish that revealed a role of the GTPase vessel sprouting [50].

\section{Concluding Remarks}

A multitude of TJ proteins have been identified and information about their functional properties is starting to become available. However, how the different junctional components cooperate to regulate the complex processes underlying cell dynamics and tissue remodelling is still poorly understood. This is best illustrated in the gap of knowledge about how transmembrane proteins regulate and are regulated by the cytoplasmic plaque. To fill such gaps will not only require detailed molecular and structural work using different model systems in vitro, it will also require more detailed in vivo studies that make use of cell type-specific inactivation of genes in different model organisms and to analyse such models under distinct physiological and pathological conditions combined with an analysis of the induced changes on a molecular, cellular, tissue, and organ level. Another emerging research area relevant for tissue remodeling is to disclose the molecalur mechanisms involved in the cross-talk between tight junctions with other cell-cell and extracellular adhesion complexes and how such processes coordinate dynamic remodeling of adhesion complexes.

\section{Acknowledgments}

Our laboratories are supported by the BBSRC (BB/N001133/1; BB/L007584/1; BB/J015032/1), Fight for Sight (1393 and 1740/1741), Special Trustees of Moorfield Eye Hospital (ST 1507 I), and the Wellcome Trust (201057/Z/16/Z).

\section{References}

\section{Van Itallie CM, Anderson JM: Architecture of tight junctions and principles of molecular} composition. Semin Cell Dev Biol 2014, 36:157-165.

2. Zihni C, Balda MS, Matter K: Signalling at tight junctions during epithelial differentiation and microbial pathogenesis. J Cell Sci 2014, 127:3401-3413. 
7

3. Kitajiri S, Katsuno T, Sasaki H, Ito J, Furuse M, Tsukita S: Deafness in occludin-deficient mice with dislocation of tricellulin and progressive apoptosis of the hair cells. Biol Open 2014, 3:759-766.

* Establishes an in vivo occludin-tricellulin functional relationship to be involved in deafness due to the regulation of hair cell function

4. Oda Y, Otani T, Ikenouchi J, Furuse M: Tricellulin regulates junctional tension of epithelial cells at tricellular contacts through Cdc42. J Cell Sci 2014, 127:4201-4212.

5. Steed E, Elbediwy A, Vacca B, Dupasquier S, Hemkemeyer SA, Suddason T, Costa AC, Beaudry JB, Zihni C, Gallagher E, et al.: MarvelD3 couples tight junctions to the MEKK1-JNK pathway to regulate cell behavior and survival. $J$ Cell Biol 2014, 204:821-838.

* Elucidation of a mechanism connecting tight junctions and JNK signalling that regulates the remodeling of the actin cytoskeleton, epithelial migration, and cellular stress response.

6. Tamura A, Tsukita S: Paracellular barrier and channel functions of $\mathbf{T J}$ claudins in organizing biological systems: advances in the field of barriology revealed in knockout mice. Seminars in cell \& developmental biology 2014, 36:177-185.

7. Kage H, Flodby P, Gao D, Kim YH, Marconett CN, DeMaio L, Kim KJ, Crandall ED, Borok Z: Claudin 4 knockout mice: normal physiological phenotype with increased susceptibility to lung injury. Am J Physiol Lung Cell Mol Physiol 2014, 307:L524-536.

* Demonstrates that claudin 4 has little effect on normal lung physiology but may function to protect against acute lung injury.

8. Nishida M, Yoshida M, Nishiumi S, Furuse M, Azuma T: Claudin-2 regulates colorectal inflammation via myosin light chain kinase-dependent signaling. Dig Dis Sci 2013, 58:1546-1559.

9. Ding L, Lu Z, Foreman O, Tatum R, Lu Q, Renegar R, Cao J, Chen YH: Inflammation and disruption of the mucosal architecture in claudin-7-deficient mice. Gastroenterology 2012, 142:305-315.

10. LaFemina MJ, Sutherland KM, Bentley T, Gonzales LW, Allen L, Chapin CJ, Rokkam D, Sweerus KA, Dobbs LG, Ballard PL, et al.: Claudin-18 deficiency results in alveolar barrier dysfunction and impaired alveologenesis in mice. Am J Respir Cell Mol Biol 2014, 51:550558.

* Demonstrates that claudin-18 regulates normal lung morphogenesis and physiology, and protects against lung injury.

11. Kim HY, Alarcon C, Pourteymour S, Wergedal JE, Mohan S: Disruption of claudin-18 diminishes ovariectomy-induced bone loss in mice. Am J Physiol Endocrinol Metab 2013, 304:E531-537.

12. Linares GR, Brommage R, Powell DR, Xing W, Chen ST, Alshbool FZ, Lau KH, Wergedal JE, Mohan S: Claudin 18 is a novel negative regulator of bone resorption and osteoclast differentiation. Journal of bone and mineral research : the official journal of the American Society for Bone and Mineral Research 2012, 27:1553-1565.

13. Sugawara T, Iwamoto N, Akashi M, Kojima T, Hisatsune J, Sugai M, Furuse M: Tight junction dysfunction in the stratum granulosum leads to aberrant stratum corneum barrier function in claudin-1-deficient mice. J Dermatol Sci 2013, 70:12-18.

14. Luissint AC, Nusrat A, Parkos CA: JAM-related proteins in mucosal homeostasis and inflammation. Semin Immunopathol 2014, 36:211-226.

15. Chatterjee S, Wang Y, Duncan MK, Naik UP: Junctional adhesion molecule-A regulates vascular endothelial growth factor receptor-2 signaling-dependent mouse corneal wound healing. PLoS One 2013, 8:e63674. 
16. Schmitt MM, Megens RT, Zernecke A, Bidzhekov K, van den Akker NM, Rademakers T, van Zandvoort MA, Hackeng TM, Koenen RR, Weber C: Endothelial junctional adhesion molecule-a guides monocytes into flow-dependent predilection sites of atherosclerosis. Circulation 2014, 129:66-76.

* Identifies endothelial JAM-A as an effector to direct inflammatory cell entry at sites of disturbed flow in large arteries where atherosclerosis develops.

17. Karshovska E, Zhao Z, Blanchet X, Schmitt MM, Bidzhekov K, Soehnlein O, von Hundelshausen P, Mattheij NJ, Cosemans JM, Megens RT, et al.: Hyperreactivity of junctional adhesion molecule A-deficient platelets accelerates atherosclerosis in hyperlipidemic mice. Circ Res 2015, 116:587-599.

* Demonstrates that JAM-A deletion in platelets leads to an increase of plaque formation and inflammatory activities, particularly in early stages of the disease.

18. Kallewaard NL, Zhang L, Chen JW, Guttenberg M, Sanchez MD, Bergelson JM: Tissue-specific deletion of the coxsackievirus and adenovirus receptor protects mice from virus-induced pancreatitis and myocarditis. Cell Host Microbe 2009, 6:91-98.

19. Mirza M, Pang MF, Zaini MA, Haiko P, Tammela T, Alitalo K, Philipson L, Fuxe J, Sollerbrant K: Essential role of the coxsackie- and adenovirus receptor (CAR) in development of the lymphatic system in mice. PLoS One 2012, 7:e37523.

20. Balda MS, Matter K: Tight junctions and the regulation of gene expression. Biochim Biophys Acta 2009, 1788:761-767.

21. Gonzalez-Mariscal L, Dominguez-Calderon A, Raya-Sandino A, Ortega-Olvera JM, Vargas-Sierra $\mathrm{O}$, Martinez-Revollar G: Tight junctions and the regulation of gene expression. Semin Cell Dev Biol 2014, 36:213-223.

22. Terry S, Nie M, Matter K, Balda MS: Rho signaling and tight junction functions. Physiology (Bethesda) 2010, 25:16-26.

23. Quiros M, Nusrat A: RhoGTPases, actomyosin signaling and regulation of the epithelial Apical Junctional Complex. Semin Cell Dev Biol 2014, 36:194-203.

24. McCormack J, Welsh NJ, Braga VM: Cycling around cell-cell adhesion with Rho GTPase regulators. J Cell Sci 2013, 126:379-391.

25. Tsapara A, Luthert P, Greenwood J, Hill CS, Matter K, Balda MS: The RhoA activator GEF$\mathrm{H1} / \mathrm{Lfc}$ is a transforming growth factor-beta target gene and effector that regulates alphasmooth muscle actin expression and cell migration. Mol Biol Cell 2010, 21:860-870.

26. Zhou Z, Guo F, Dou Y, Tang J, Huan J: Guanine nucleotide exchange factor-H1 signaling is involved in lipopolysaccharide-induced endothelial barrier dysfunction. Surgery 2013, 154:621-631.

27. Tian Y, Mambetsariev I, Sarich N, Meng F, Birukova AA: Role of microtubules in attenuation of PepG-induced vascular endothelial dysfunction by atrial natriuretic peptide. Biochim Biophys Acta 2015, 1852:104-119.

28. Nie M, Aijaz S, Leefa Chong San IV, Balda MS, Matter K: The Y-box factor ZONAB/DbpA associates with GEF-H1/Lfc and mediates Rho-stimulated transcription. EMBO Rep 2009, 10:1125-1131.

29. Nie M, Balda MS, Matter K: Stress- and Rho-activated ZO-1-associated nucleic acid binding protein binding to $\mathbf{p} 21$ mRNA mediates stabilization, translation, and cell survival. Proc Natl Acad Sci U S A 2012, 109:10897-10902.

30. Terry SJ, Zihni C, Elbediwy A, Vitiello E, Leefa Chong San IV, Balda MS, Matter K: Spatially restricted activation of RhoA signalling at epithelial junctions by p114RhoGEF drives junction formation and morphogenesis. Nat Cell Biol 2011, 13:159-166. 
9

31. Elbediwy A, Zihni C, Terry SJ, Clark P, Matter K, Balda MS: Epithelial junction formation requires confinement of Cdc42 activity by a novel SH3BP1 complex. J Cell Biol 2012, 198:677-693.

32. Nakajima $H$, Tanoue T: Lulu2 regulates the circumferential actomyosin tensile system in epithelial cells through p114RhoGEF. The Journal of cell biology 2011, 195:245-261.

33. Itoh M, Tsukita S, Yamazaki Y, Sugimoto H: Rho GTP exchange factor ARHGEF11 regulates the integrity of epithelial junctions by connecting ZO-1 and RhoA-myosin II signaling. Proc Natl Acad Sci U S A 2012, 109:9905-9910.

34. Chang YJ, Pownall S, Jensen TE, Mouaaz S, Foltz W, Zhou L, Liadis N, Woo M, Hao Z, Dutt P, et al.: The Rho-guanine nucleotide exchange factor PDZ-RhoGEF governs susceptibility to diet-induced obesity and type 2 diabetes. eLife 2015, 4.

35. Qin Y, Meisen WH, Hao Y, Macara IG: Tuba, a Cdc42 GEF, is required for polarized spindle orientation during epithelial cyst formation. J Cell Biol 2010, 189:661-669.

36. Guillemot L, Paschoud S, Jond L, Foglia A, Citi S: Paracingulin regulates the activity of Rac1 and RhoA GTPases by recruiting Tiam1 and GEF-H1 to epithelial junctions. Mol Biol Cell 2008, 19:4442-4453.

37. Guillemot L, Guerrera D, Spadaro D, Tapia R, Jond L, Citi S: MgcRacGAP interacts with cingulin and paracingulin to regulate Rac1 activation and development of the tight junction barrier during epithelial junction assembly. Mol Biol Cell 2014, 25:1995-2005.

38. Rodgers LS, Beam MT, Anderson JM, Fanning AS: Epithelial barrier assembly requires coordinated activity of multiple domains of the tight junction protein ZO-1. J Cell Sci 2013, 126:1565-1575.

39. Fanning AS, Van Itallie CM, Anderson JM: Zonula occludens-1 and -2 regulate apical cell structure and the zonula adherens cytoskeleton in polarized epithelia. Mol Biol Cell 2012, 23:577-590.

40. Tokuda S, Higashi T, Furuse M: ZO-1 knockout by TALEN-mediated gene targeting in MDCK cells: involvement of ZO-1 in the regulation of cytoskeleton and cell shape. PLoS One 2014, 9:e104994.

41. Tornavaca O, Chia M, Dufton N, Almagro LO, Conway DE, Randi AM, Schwartz MA, Matter K, Balda MS: ZO-1 controls endothelial adherens junctions, cell-cell tension, angiogenesis, and barrier formation. $J$ Cell Biol 2015, 208:821-838.

* Identifies the molecular mechanisms by which ZO-1 regulates endothelial actin cytoskeleton, migration, angiogenesis, and barrier formation and its cross-talk with VE-cadherindependent cell-cell tension.

42. Lynch AM, Hardin J: The assembly and maintenance of epithelial junctions in C. elegans. Front Biosci (Landmark Ed) 2009, 14:1414-1432.

43. Choi W, Jung KC, Nelson KS, Bhat MA, Beitel GJ, Peifer M, Fanning AS: The single Drosophila ZO-1 protein Polychaetoid regulates embryonic morphogenesis in coordination with Canoe/afadin and Enabled. Mol Biol Cell 2011, 22:2010-2030.

44. Tepass U: The apical polarity protein network in Drosophila epithelial cells: regulation of polarity, junctions, morphogenesis, cell growth, and survival. Annu Rev Cell Dev Biol 2012, 28:655-685.

45. Norden PR, Kim DJ, Barry DM, Cleaver OB, Davis GE: Cdc42 and k-Ras Control Endothelial Tubulogenesis through Apical Membrane and Cytoskeletal Polarization: Novel Stimulatory Roles for GTPase Effectors, the Small GTPases, Rac2 and Rap1b, and Inhibitory Influence of Arhgap31 and Rasa1. PLoS One 2016, 11:e0147758. 
10

46. Whiteman EL, Fan S, Harder JL, Walton KD, Liu CJ, Soofi A, Fogg VC, Hershenson MB, Dressler GR, Deutsch GH, et al.: Crumbs3 is essential for proper epithelial development and viability. Mol Cell Biol 2014, 34:43-56.

* Characterisation of Crumbs 3 as regulator of epithelial morphogenesis by linking the apical membrane to the underlying ezrin-containing cytoskeleton.

47. Varelas X, Samavarchi-Tehrani P, Narimatsu M, Weiss A, Cockburn K, Larsen BG, Rossant J, Wrana JL: The Crumbs complex couples cell density sensing to Hippo-dependent control of the TGF-beta-SMAD pathway. Dev Cell 2010, 19:831-844.

48. Zihni C, Munro PM, Elbediwy A, Keep NH, Terry SJ, Harris J, Balda MS, Matter K: Dbl3 drives

Cdc42 signaling at the apical margin to regulate junction position and apical differentiation. J Cell Biol 2014, 204:111-127.

* Elucidation of the molecular mechanism that drives apical $\mathrm{Cdc42}$ activation during apical differentiation and domain size specification.

49. Ngok SP, Geyer R, Liu M, Kourtidis A, Agrawal S, Wu C, Seerapu HR, Lewis-Tuffin LJ, Moodie KL, Huveldt D, et al.: VEGF and Angiopoietin-1 exert opposing effects on cell junctions by regulating the Rho GEF Syx. J Cell Biol 2012, 199:1103-1115.

50. Wu C, Agrawal S, Vasanji A, Drazba J, Sarkaria S, Xie J, Welch CM, Liu M, Anand-Apte B, Horowitz A: Rab13-dependent trafficking of RhoA is required for directional migration and angiogenesis. $J$ Biol Chem 2011, 286:23511-23520.

Note: We appologize to authors of original papers published before 2009 as we were not able to reference them due to journal reference number restrictions. 

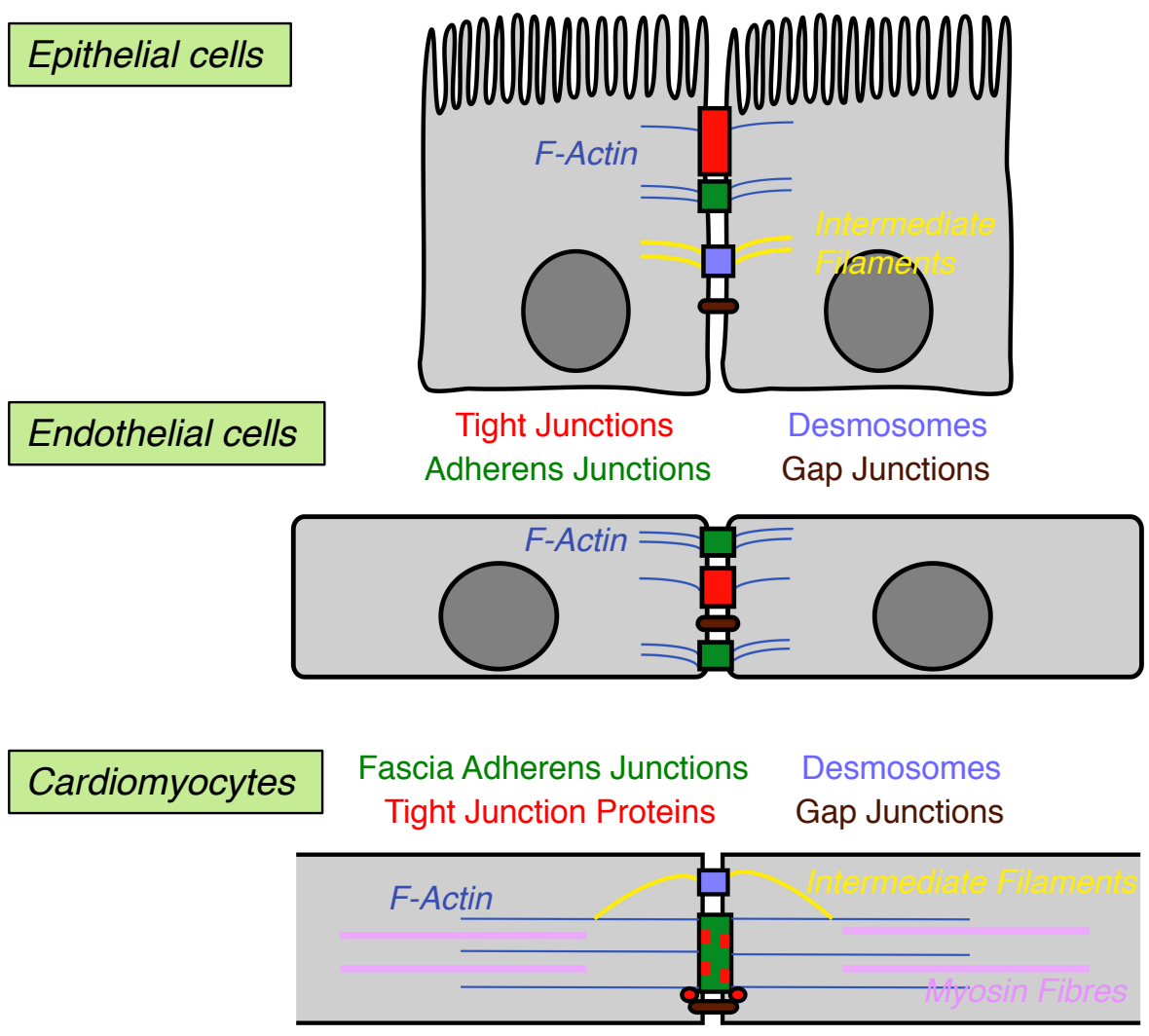

Figure 1. Intercellular junctions in epithelia, endothelia and cardiomyocytes.

Schematic representation of main intercellular junctions in vertebrate cell types discussed in this review. Epithelia and endothelia form tight and adherens junctions that are often closely associated and linked to the actin cytoskeleton. Mature tight and adherens junctions are morphologically and biochemically distinct but form from a primordial junction that contains tight and adherens junctions. In epithelia, tight junctions generally form the apical/lateral border and, hence, are localized more apically than adherens junctions. In endothelia, the two junctions can be intercalated. Cardiomyocytes do not form tight junctions but form a variant of adherens junctions, fascia adherens junctions, that also contains proteins associated with tight junctions in epithelia. Gap junctions, which form small channels allowing cell-cell communication, are expressed in all three cell types and also recruite tight junction proteins in some cell types. Epithelia and cardiomyocites also form desmosomes that link the junctional complex to intermediate filaments. 


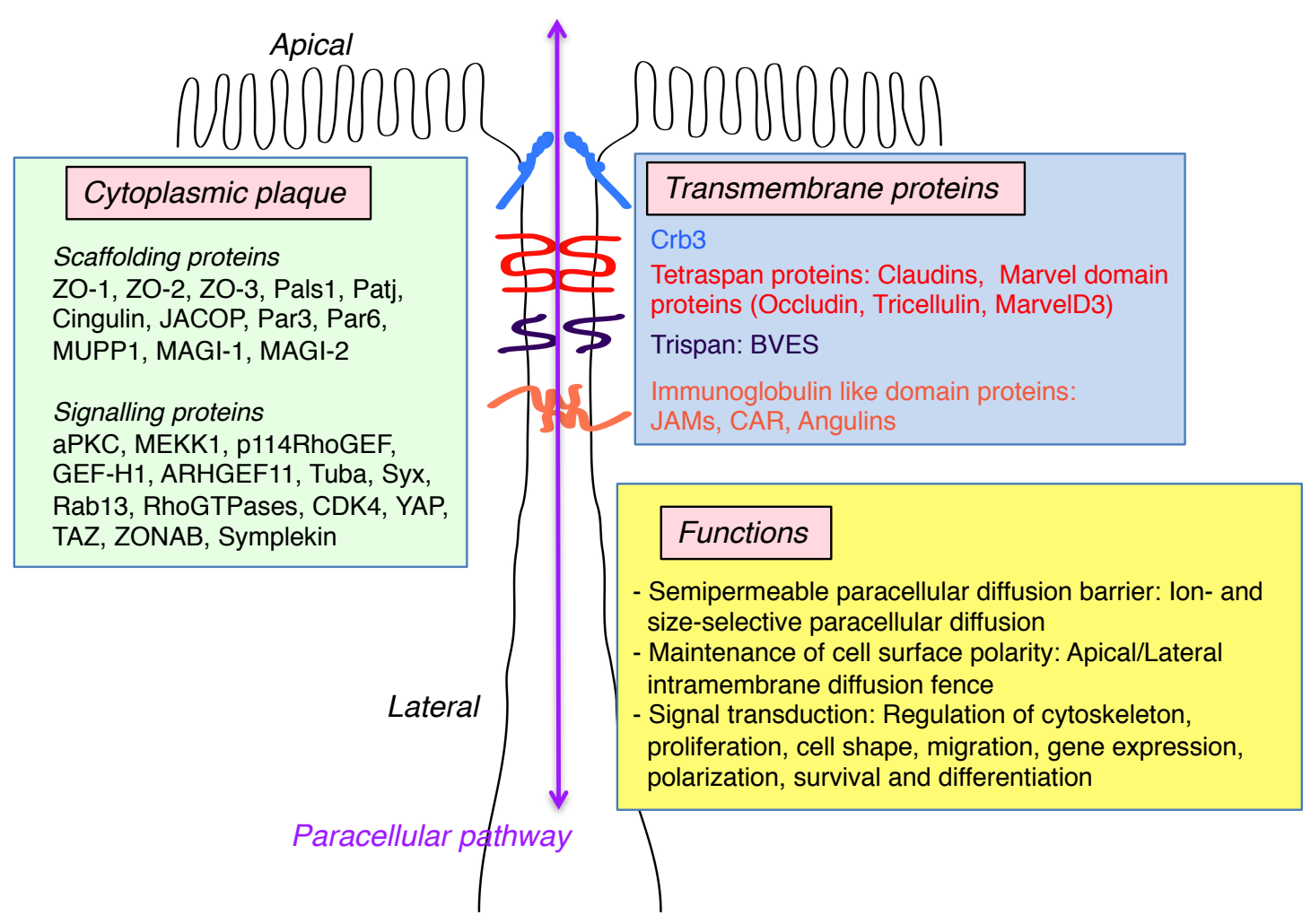

Figure 2. Composition and function of tight junctions.

Tight junctions are formed by a heterogenous set of transmembrane proteins that includes the tetraspan proteins of the claudin family and three Marvel domain proteins, as well as BVES, adhesion proteins with immunoglobulin-like domains, and the polarity signalling protein Crb3. These transmembrane proteins interact with a complex protein web, the cytoplasmic plaque, formed by scaffolding and signalling proteins that links the junction to the actin cytoskeleton. Tight junctions have three main functions. Firstly, they form paracellular diffusion barriers that enable epithelia and endothelia to form tissue barriers; these barriers are semipermeable and allow size- and ion-selective paracellular diffusion of solutes. Secondly, tight junctions form an intramembrane diffusion barrier that restricts the intermixing of apical and lateral plasma membrane components. Thirdly, tight junctions contain a complex set of signalling proteins that are components of regulatory mechanisms that guide cell behaviour and differentiation. Note, only proteins discussed in this review are indicated. 